\title{
EFECTIVIDAD DE UN PROGRAMA EDUCATIVO SOBRE VENTA DIRECTA DE UN PRODUCTO EN EL NIVEL DE CONOCIMIENTO EN MADRES DE FAMILIA DE LA I.E. TORIBIO POLO - ICA, ENERO 2011
}

\section{Program educational effectiveness of a direct selling on a product in the level of knowledge in mothers of} family IE Toribio Polo - Ica, January 2011

Nelyda Salvatierra Oyola ${ }^{1, a, b, c}$

${ }^{1}$ Facultad de Enfermería. Universidad Nacional San Luis Gonzaga de Ica. Ica, Perú.

${ }^{a}$ Licenciada en Enfermería, ${ }^{\mathrm{b}}$ Especialista en Clínica Pediátrica, ${ }^{\mathrm{c}}$ Magíster en Administración.

\section{RESUMEN}

Objetivo: Determinar la efectividad del programa educativo sobre venta directa de un producto en el nivel de conocimiento de las madres de familia de la I.E. Toribio Polo, Enero 2011. Material y métodos: El tipo de estudio corresponde al cuantitativo, de diseño cuasi experimental, prospectivo; la población de estudio estuvo constituida por 840 madres de estudiantes de la Institución Educativa Toribio Polo, de las cuales se tomó por muestreo no probabilístico por conveniencia a 20 madres, las cuales participaron de un programa educativo sobre venta directa de un producto, utilizando como técnica la encuesta y como instrumento un cuestionario aplicado en dos oportunidades para evaluar el nivel de conocimientos antes y después del programa educativo y comprobar la efectividad del mismo. Dicho instrumento fue validado a través de juicio de expertos y su confiabilidad se demostró a través del alfa de Cronbach. Resultados: Las características sociodemográficas de las madres de familia en estudio correspondieron a 28 y 36 años (50\%); grado de instrucción secundaria (45\%); tipo de familia nuclear (55\%) teniendo como ocupación ama de casa $(60 \%)$; respecto a la efectividad del programa educativo fue efectivo demostrado a través de la prueba $T$ de Student $t>1.725(t=6.44)$ luego de haber evidenciado la diferencia entre los niveles de conocimientos alcanzados en el pre test (nivel bajo: 70\%) y post test (nivel alto: 90\%). Conclusiones: El programa educativo sobre venta directa de un producto fue efectivo en las madres de familia de la I.E. Toribio Polo Enero 2011.

PALABRAS CLAVE : Programa educativo, efectividad, conocimiento, venta directa, producto.

\section{SUMMARY}

Objectives: to determine the effectiveness of the educational program on direct sale of a product on the level of knowledge of the mothers of the IE Toribio Polo, January 2011 Material and Methods: The study is the type of quantitative, quasi-experimental design, Prospective, the study population consisted of 840 mothers of students of the Toribio Polo School, which was taken by non-probabilistic convenience sampling to 20 mothers who participated in an educational program on direct sale of a product, using art as an instrument survey and a questionnaire administered on two occasions to assess the level of knowledge before and after the educational program and verify 
its effectiveness. The instrument was validated through expert opinion and reliability was demonstrated by Cronbach's alpha. Results: The sociodemographic characteristics of the mothers studied corresponded to 28 and 36 years (50\%) secondary education level (45\%), nuclear family type $(55 \%)$ having as occupation housewife $(60 \%)$ regarding the effectiveness of the educational program was proven effective through the Student t-test $p>1725(p=3.52)$ after having shown the difference between the levels of knowledge gained in the pretest (low: 70\%) and post test (high: 90\%). Conclusions: The educational program on direct sale of a product was effective in the mothers of the IE Toribio Polo Ica January 2011.

KEYWORDS: Educational program, effectiveness, knowledge, direct sales, product.

\section{INTRODUCCIÓN}

La venta directa, también conocida como comercialización de bienes de consumo y servicios directamente a los consumidores, tiene como base el contacto personal de un vendedor o vendedora independiente fuera de un local comercial, se ha convertido hoy en día en una estrategia de Marketing, así como también una opción de desarrollo económico en cualquier tipo de persona que decida optar dicha práctica. Las empresas de Venta Directa siguen creciendo en volumen y facturación alrededor del mundo, también este fenómeno ocurre en Latinoamérica pese al colapso que varias economías están viviendo como las de Venezuela, Argentina y Uruguay fundamentalmente. Es así que la Venta Directa parece fortalecerse más y más en momentos difíciles, de crisis $y$ depresiones. Tal vez sea porque el objetivo comparte con el sistema de comercialización, emociones que van más allá de las remuneraciones que puedan obtener a partir de sus ventas. Hoy en día frente a la igualdad de posibilidades, oportunidades que hay entre las mujeres y los varones, son necesarias mayores oportunidades laborales, siendo en la mayoría de países en vías de desarrollo, el trabajo independiente. Las organizaciones de Venta Directa como Avon Cosmetics, Omnilife y otras que operan en nuestro continente con éxito desde hace muchos años, cuentan con verdaderos "ejércitos" como el de Omnilife de México con casi tres millones de distribuidores bajo la conducción de un líder de vendedoras y vendedores que hacen frente al día a día con éxito, sean cual sean las dificultades que el mercado les presenta. Según Arboleda en su estudio de factibilidad para la creación de una empresa consultora en Gestión Empresarial, quien realizó investigación de mercado a las empresas de la ciudad de Quito para determinar los gustos y preferencias relacionadas con el servicio de consultoría en Gestión Empresarial, así como los factores vinculados con el precio $y$ distribución más adecuada para el consumidor. Se efectuó el estudio técnico que determinó las variables de los requerimientos técnicos y organizacionales necesarios para 
que la empresa desarrolle sus actividades en una muestra de 378 empresarios, utilizando como técnica la encuesta y entrevista. De acuerdo a la evaluación realizada se obtuvo que contratan capacitación para su personal "Casi Siempre" en un $43,12 \%$ de las empresas que contratan capacitación, el $52,38 \%$ prefieren cursos (1).

Castuera et al., realizaron una encuesta sobre Plan de Negocios para la creación de una Microfinanciera en Apoyo a la Mujer Emprendedora en Puebla, México, en la cual se aplicó una encuesta con los puntos incluidos en los constructores del plan; obteniéndose los siguientes resultados: La edad de las mujeres encuestadas osciló entre los 21 y los 45 años, un $72 \%$ de las mujeres estaban dispuestas a adquirir un crédito después de la educación impartida, existiendo un $43 \%$ que nunca había solicitado un crédito para negocios, a pesar de tener sólo un $25 \%$ que ya contaba con un negocio, existía un $75 \%$ que no contaba con un negocio pero que tenía el entusiasmo y decisión para hacerlo(2).

Armas realizó un estudio explicativo transaccional del campo cuantitativo sobre el marketing y su influencia en las ventas en las microempresas en el Perú. la investigación tuvo como finalidad determinar el impacto del marketing sobre las ventas en las microempresas del Distrito de Pueblo Nuevo, para efectos de difundir el grado de conocimiento que poseían los microempresarios de dicho distrito, la frecuencia con que se usaba esta técnica en el giro de los pequeños negocios y los

resultados que se obtienen sobre las ventas como consecuencia de la utilización del marketing. El tipo de diseño fue no experimental, habiéndose desarrollado bajo un paradigma cuantitativo; la población estuvo conformada por 20 microempresas, la técnica utilizada fue el cuestionario estructurado, los resultados arrojaron que el marketing en la mayoría de los casos posee un impacto positivo sobre las ventas (3).

El desconocimiento sobre Marketing básico y estrategias respecto al mismo, imperan en cada evento de fracaso empresarial, además la existencia de una economía deficiente en nuestro país, es una las debilidades más importantes que aún no se supera, sin embargo la creatividad en microempresas y oportunidades de venta directa de un producto persisten, es así como describimos la necesidad de capacitar respecto a aspectos básicos de la venta directa de un producto, como parte de la solución a la crisis económica por el ingreso deficiente que no logra cubrir los gastos generados por la canasta familiar y que en el caso de las madres de familias, sin necesidad de salir de sus hogares podrían generar ingresos favorables a partir de dicha estrategia.

\section{MATERIAL Y METODOS}

De acuerdo a la presentación de los resultados en tablas y gráficos, la investigación corresponde a una investigación cuantitativa. Es una investigación de nivel aplicativo, cuyo instrumento de aplicación es el programa educativo dirigido a una muestra de estudio. Corresponde a los estudios cuasi 
experimentales, porque manipula una de las variables, la independiente para obtener una respuesta deseada.

La población de estudio está conformada por todas las madres de familia de la I.E. Toribio Polo - Ica, la cual corresponde a 840; sin embargo por criterio del investigador, se toma en cuenta el muestreo intencional, teniendo en cuenta para la muestra 20 madres de familia que aceptaron y afirmaron un compromiso de participar de la investigación desde el inicio al final del mismo con instrucción primaria, con deseos de incursionar en las ventas, entre las edades de 20 a 50 años de edad. Cuya ocupación sea ama de casa y/o independientes que dispongan de tiempo. En la recolección de datos se utilizó la técnica de la encuesta, considerando además para la aplicación del programa educativo diferentes metodologías comprendidas en el mismo programa. Como instrumento se utilizó un cuestionario de opción múltiple el cual se sometió a juicio de expertos previamente y se confirmó su confiabilidad a través del Alfa de Crombach. Dicho instrumento se aplicó en dos oportunidades, Pre y Post Test Una vez recogidos los datos se procedió a organizar y tabular la información, luego empleando el programa SPSS15 (Paquete estadístico para la investigación en ciencias sociales) Para el procesamiento y análisis de datos: clasificación de datos, tabulación de datos y luego se realizo la interpretación de los mismos.

Se utilizó la estadística descriptiva e inferencial para el análisis respectivo. Para establecer los niveles de conocimientos se tomó en cuenta la escala de Stanones usando promedio y desviación estándar. La efectividad del programa educativo se demostró mediante el estadístico $\mathrm{T}$ de Student.

\section{RESULTADOS}

En la distribución de las madres de familia según datos demográficos se encuentra que las edades corresponde en mayor proporción de 28-36 años alcanzando un 50\%(10 madres); el grado de instrucción fue secundaria en el 45\%(9 madres) de la muestra, el tipo de familia fue nuclear en el 55\%(11 madres) siendo la ocupación ama de casa en el 60\%(12 madres) de la muestra.

El nivel de conocimiento según conceptos básicos del marketing se obtuvo en el pre-test mayor predominio en el nivel medio y bajo alcanzando en cada uno 40\%(8 madres); sin embargo en el post -test alcanzó un 100\%(20 madres) el nivel alto. El nivel de conocimiento según las cuatro $P$ del marketing se obtuvo en el pre-test mayor con predominio en el nivel medio alcanzando 60\%(12 madres), sin embargo en el post -test alcanzó un 100\%(20 madres) el nivel alto.

El nivel de conocimiento según perfil del vendedor se obtuvo en el pre-test mayor predominio en el nivel bajo alcanzando 80\%(16 madres); sin embargo en el post -test alcanzó un 80\%(16 madres) en el nivel alto.

El nivel de conocimiento según conceptos básicos del marketing se obtuvo en el pre-test mayor predominio en el nivel bajo alcanzando 70\%(14 madres); sin embargo en el post -test alcanzó un 90\%(18 madres) el nivel alto. 
El nivel de conocimiento sobre venta de un producto sufrió una variación entre el pre-test y post -test observándose una tendencia favorable evidenciado por el incremento en el

Tabla 1.Datos sociodemográficos de las madres de familia de la i.e. Toribio Polo. ENERO 2011

\begin{tabular}{lrr}
\hline Edad & $\mathbf{f}$ & $\mathbf{\%}$ \\
\hline $\mathbf{2 8 - 3 6}$ & 10 & $50 \%$ \\
$\mathbf{3 7 - 4 5}$ & 4 & $20 \%$ \\
$\mathbf{4 6 - 5 4}$ & 6 & $30 \%$ \\
\hline Total & 20 & $100 \%$ \\
\hline Grado instrucción & $\mathrm{f}$ & $\%$ \\
\hline Primaria & 6 & $30 \%$ \\
& & \\
\hline Secundaria & 9 & $45 \%$ \\
\hline Superior técnica & 5 & $25 \%$ \\
\hline Total & 20 & $100 \%$ \\
\hline Tipo de familia & $\mathrm{f}$ & $\%$ \\
\hline Nuclear & 11 & $55 \%$ \\
\hline Extensa & 3 & $15 \%$ \\
\hline Reconstituida & 1 & $5 \%$ \\
\hline Monoparental & 5 & $25 \%$ \\
\hline Total & 20 & $100 \%$ \\
\hline Ocupación & $\mathrm{f}$ & $\%$ \\
\hline Independiente & 8 & $40 \%$ \\
(comerciante & & \\
informal) & & \\
\hline Su casa & 12 & $60 \%$ \\
\hline Total & 20 & $100 \%$ \\
\hline
\end{tabular}

Tabla 4. Nivel de conocimiento sobre venta directa de un producto según perfil del vendedor ENERO 2011

\begin{tabular}{cccccc}
\hline $\begin{array}{c}\text { PRE } \\
\text { TEST }\end{array}$ & $\mathbf{F}$ & $\%$ & $\begin{array}{r}\text { POST } \\
\text { TEST }\end{array}$ & F & $\%$ \\
\hline ALTO & 0 & $0 \%$ & ALTO & 16 & $80 \%$ \\
MEDIO & 4 & $20 \%$ & MEDIO & 4 & $20 \%$ \\
BAJO & 16 & $80 \%$ & BAJO & 0 & $0 \%$ \\
TOTAL & 20 & $100 \%$ & TOTAL & 20 & $100 \%$ \\
\hline
\end{tabular}

nivel alto. Lo cual confirma la adquisición de conocimientos respecto a la venta de un producto.

Tabla 2. Nivel de conocimiento sobre venta directa de un producto según conceptos básicos del marketing Toribio Polo. ENERO 2011

\begin{tabular}{cccccc}
\hline $\begin{array}{c}\text { PRE } \\
\text { TEST }\end{array}$ & $\mathbf{F}$ & $\%$ & $\begin{array}{c}\text { POST } \\
\text { TEST }\end{array}$ & $\mathbf{F}$ & $\%$ \\
\hline ALTO & 4 & $20 \%$ & ALTO & 20 & $100 \%$ \\
MEDIO & 8 & $40 \%$ & MEDIO & 0 & $0 \%$ \\
BAJO & 8 & $40 \%$ & BAJO & 0 & $0 \%$ \\
TOTAL & 20 & $100 \%$ & TOTAL & 20 & $100 \%$ \\
\hline
\end{tabular}

Tabla 3. Nivel de conocimiento sobre venta directa de un producto según las cuatro $\mathrm{P}$ del marketing ENERO 2011

\begin{tabular}{cccccc}
\hline $\begin{array}{c}\text { PRE } \\
\text { TEST }\end{array}$ & F & $\%$ & $\begin{array}{c}\text { POST } \\
\text { TEST }\end{array}$ & F & $\%$ \\
\hline ALTO & 0 & $0 \%$ & ALTO & 20 & $100 \%$ \\
MEDIO & 12 & $60 \%$ & MEDIO & 0 & $0 \%$ \\
BAJO & 8 & $40 \%$ & BAJO & 0 & $0 \%$ \\
TOTAL & 20 & $100 \%$ & TOTAL & 20 & $100 \%$ \\
\hline
\end{tabular}

Tabla 5. Nivel de conocimiento sobre venta directa de un producto en madres de familia de la I. E. Toribio Polo Ica. ENERO 2011

\begin{tabular}{cccccc}
\hline $\begin{array}{c}\text { PRE } \\
\text { TEST }\end{array}$ & $\mathbf{F}$ & $\%$ & $\begin{array}{r}\text { POST } \\
\text { TEST }\end{array}$ & $\mathbf{F}$ & $\%$ \\
\hline ALTO & 2 & $10 \%$ & ALTO & 18 & $90 \%$ \\
MEDIO & 4 & $20 \%$ & MEDIO & 2 & $10 \%$ \\
BAJO & 14 & $70 \%$ & BAJO & 0 & $0 \%$ \\
TOTAL & 20 & $100 \%$ & TOTAL & 20 & $100 \%$ \\
\hline
\end{tabular}




\section{DISCUSIÓN}

La venta directa de un producto se ha constituido en una alternativa de sobrevivencia en nuestro medio, en ocasiones su desarrollo sin el conocimiento previo de los estándares y conceptos básicos del marketing provocan el desconcierto en la persona que lo ejecuta y entre otros comerciantes, siendo necesario educar y capacitar en torno a alternativas de solvencia económica bien direccionadas como alternativa para muchas mujeres que en muchos casos se constituyen como jefe de familias.

En el estudio realizado observamos la distribución de las madres de familia según datos demográficos encontrándose que la edad corresponde en mayor proporción a 2836 años alcanzando un 50\%; el grado de instrucción fue secundaria en el $45 \%$ de la muestra, el tipo de familia fue nuclear en el $55 \%$ siendo la ocupación ama de casa en el $60 \%$ de la muestra. Como se puede observar las madres corresponden a una etapa adulta joven capaces de contar con la maduración y responsabilidad para ejercer labores de venta considerando además el aporte económico a sus hogares en torno a su familia o haciéndose responsables de los mismos. Tomando en cuenta que la mayoría tienen instrucción secundaria constituiría una fortaleza para el aprendizaje en torno al marketing en sus aspectos básicos. Los resultados guardan cierta coherencia con el estudio confirmándose la factibilidad de trabajo como parte de su desarrollo personal y económico con dicho grupo de interés.
El nivel de conocimiento como variable de estudio se identificó en dos momentos, antes y después de la aplicación del programa educativo, asimismo cada dimensión sirvió para establecer los gráficos y evidenciar mejor los resultados, tal es así que según conceptos básicos del marketing se obtuvo en el pre-test mayor predominio en el nivel medio y bajo alcanzando en cada uno $40 \%$; sin embargo en el post -test alcanzó un 100\% el nivel alto. Según la dimensión considerada como: Las cuatro $\mathrm{P}$ del marketing se obtuvo en el pre-test mayor predominio en el nivel medio alcanzando 60\%; sin embargo en el post -test alcanzó un $100 \%$ el nivel alto. Según perfil del vendedor se obtuvo en el pretest mayor predominio en el nivel bajo alcanzando $80 \%$; sin embargo en el post -test alcanzó un $80 \%$ en el nivel alto. Arboleda JC. (2010) De acuerdo a la evaluación realizada se obtuvo que contratan capacitación para su personal "casi siempre" en un $43,12 \%$; de las empresas que contratan capacitación, el $52,38 \%$ prefieren cursos. Se considera que el proyecto de creación de una empresa consultora en gestión empresarial en la ciudad de Quito es factible por lo que se recomienda su implementación. ${ }^{(2)}$ Vemos que los niveles de conocimiento son deficientes antes de la aplicación del programa educativo. Notándose la efectividad de dicha actividad evidenciada por el aumento del nivel de conocimiento lo cual puede constituir una alternativa previa a microempresas.

Los resultados obtenidos reflejan que la creación y mantenimiento de una cultura emprendedora y la educación a la comunidad 
asegura el éxito empresarial si se hace el respectivo seguimiento y capacitación en pequeños empresarios, beneficiándose no solo los comprometidos directamente con la microempresa sino también da lugar a puestos de trabajos y favorece el desarrollo de la comunidad. Flores et al demostraron que la mejor forma de introducir un producto al mercado es creando un plan de marketing, no sólo para introducir un producto sino también para incrementar las ventas y otros factores, demostrando que al hacer antes el estudio de mercado, se facilitó la aceptación de los jabones naturales por parte de las mujeres en un $85 \%(5)$.

Se entiende por venta directa o venta a domicilio la comercialización fuera de un establecimiento comercial de bienes $y$ servicios directamente al consumidor (3), estos aspectos validan la necesidad de aprendizaje en torno al marketing siendo necesario tomar en cuenta las mejores estrategias de venta para el éxito del mismo. Los costes variables son aquellos que varían en función del nivel de actividad de la empresa (3). Parece claro que lo que el consumidor planea comprar no es un bien en sí mismo, sino la función o servicio que ese bien es capaz de prestar. De acuerdo con esta idea, el consumidor evalúa, compara y ordena las marcas, alternativas que forman parte de la elección en función de sus respectivos niveles de calidad, esto es, en función de sus capacidades respectivas para buscar las ventajas buscadas, generar utilidad y, solo así, producir el deseado sentimiento de bienestar general.
Puesto que no hay prestación alguna sin la correspondiente contraprestación ni la posibilidad de obtener utilidad sin pagar por ello, el individuo debe estimar si - y en qué medida las expectativas de beneficio o las ventajas que espera recibir compensan de algún modo los esfuerzos y sacrificios que necesariamente habrá de realizar.

De esta manera surge el concepto de valor percibido: como el resultado de una evaluación global del producto basado en la estimación de lo que el consumidor espera recibir a cambio de todo lo que sacrifica (dinero, tiempo, o esfuerzo físico o mental) (3), otro aspecto importante para la venta de productos lo constituye la actitud, definida como la forma de actuar de una persona, el comportamiento que emplea un individuo para hacer las cosas. En este sentido, puede considerarse como cierta forma de motivación social -de carácter, por tanto, secundario, frente a la motivación biológica, de tipo primario- que impulsa y orienta la acción hacia determinados objetivos y metas.

\section{Correspondencia:}

Mg. Nélida Salvatierra Oyola

Correo electrónico: nelydaso@hotmail.com

\section{REFERENCIAS BIBLIOGRAFICAS}

1. Arboleda JC. Estudio de factibilidad para la creación de una empresa consultora en gestión empresarial. Tesis de Grado. Ibarra, Ecuador. Universidad Técnica del Norte. 2010.

2. Castuera J, Hernández G. Plan de Negocios para la creación de una microfinanciera en apoyo a la mujer 
emprendedora. Tesis Licenciatura.

Administración de Empresas.

Departamento de Administración de

Empresas y Mercadotecnia, Escuela de

Negocios y Economía, Puebla, Mexico.

Universidad de las Américas; 2007.

3. Armas E. Marketing y su influencia en las ventas en las microempresas; Perú. 2012.

4. Cancela EM, Gasca RE. Fomento de la actividad emprendedora por el Programa Impulsa. Casos: GAM, INNOVEM y PROCAMIX de Puebla. Tesis Licenciatura. Administración de Empresas. Departamento de Administración de Empresas y Mercadotecnia, Escuela de Negocios y Economía. Puebla, Mexico. Universidad de las Américas;2006
5. Flores JD, Torres XG. Plan de Marketing para la Introducción de jabones naturales en la Ciudad de Puebla Tesis Licenciatura. Administración de Empresas. Departamento de Administración de Empresas y Mercadotecnia, Escuela de Negocios y Economía. Puebla, Mexico. Universidad de las Américas. 2004.

Recibido: 15/10/13

Aprobado para Publicación: 10/11/13 